\title{
Obesidade infantil: conhecimentos e práticas de enfermeiros da Atenção Básica*
}

Recebido em: 23/04/2012

Aceito em: 12/07/2012

\author{
Sarah Nilkece Mesquita Araújo' \\ Maria Helena Barros Araújo Luz ${ }^{2}$ \\ Silvana Santiago da Rocha ${ }^{3}$ \\ Grazielle Roberta Freitas da Silva ${ }^{4}$ \\ Marianne Rocha Duarte 5 \\ Naiana Martins de Sandes ${ }^{6}$
}

Objetivou-se, neste estudo, analisar conhecimento e práticas de enfermeiros da atenção básica sobre a obesidade infantil, com pesquisa exploratória descritiva de abordagem quantitativa com 34 enfermeiros da Estratégia de Saúde da Família (ESF), no período de fevereiro a março de 2010. A análise revelou que a maioria dos enfermeiros da ESF já teve treinamento específico em saúde da criança, porém, julgou ter conhecimento insuficiente sobre nutrição e dietética. Concluiu-se que há necessidade de aperfeiçoamento dos profissionais para lidar com o distúrbio e adoção de uma política de atenção à saúde da criança na ESF que enfoque a obesidade infantil.

Descritores: Obesidade, Enfermagem, Atenção Básica.

\section{Childhood obesity: knowledge and skills of nurses in primary care}

This study aims to evaluate knowledge and practices of primary care nurses about childhood obesity with exploratory descriptive research with a quantitative approach with 34 nurses from the Family Health Strategy (FHS) in February to March 2010.The analysis revealed that most of nurses from ESF has had contact with the child's health, but most believed to have insufficient knowledge about nutrition and diet. It was concluded that there is need for improvement of professionals to deal with the disorder and a policy of attention to child health in the ESF that focuses on childhood obesity.

Descriptors: Obesity, Nursing, Primary Health Care.

\section{La obesidad infantil: el conocimiento y las prácticas de enfermería en la atención primaria}

Este estudio tiene como objetivo evaluar los conocimientos y las prácticas de los enfermeros de atención primaria sobre la obesidad infantil, con investigación exploratoria descriptiva, con un enfoque cuantitativo con 34 enfermeras de la Estrategia de Salud Familiar (ESF) en febrero a marzo de 2010. El análisis reveló que la mayoría de las enfermeras del FSE ha tenido contacto con la salud del niño, pero creía que tenía insuficientes conocimientos acerca de la nutrición y la dieta. Se concluyó que es necesario, para la mejora de los profesionales para hacer frente a la enfermedad y, una política de atención a la salud infantil en el FSE que se centra en la obesidad infantil.

Descriptores: Obesidad, Enfermería, Atención Primaria de Salud.

\section{INTRODUÇÃO}

A obesidade infantil é definida como um excesso de gordura corporal relacionado à massa magra, e o sobrepeso como uma proporção relativa de peso maior que a desejável para a altura(1). Estudos apontam a obesidade como o problema nutricional mais prevalente nos países desenvolvidos, chegando a afetar um terço da população geral e $15 \%$ a $20 \%$ das crianças ${ }^{(3)}$. A afecção ocorre mais frequentemente no primeiro ano de vida, entre os 5 e 6 anos e na adolescência ${ }^{(2)}$, visto que essa faixa da população é, do ponto de vista psicológico, sócio-econômico e cultural, dependente do ambiente onde vive ${ }^{(4-5)}$.

A enfermagem tem um importante papel na promoção de hábitos e alimentação saudáveis, prevenção, identificação de riscos e detecção precoce da obesidade, devendo considerar a família como núcleo de atendimento, a partir de relação dialógica positiva com pais e filhos ${ }^{(6)}$. Entende-se que a Estratégia de Saúde da Família (ESF), que até então se concentra prioritariamente no combate à desnutrição infantil, precisa integrar-se nesse contexto e assimilar novos conceitos para ampliar suas ações no tocante às doenças metabólicas da infância.

1 Enfermeira. Mestranda do Programa de Pós-graduação em Enfermagem da UFPI. Especialista em Docência do Ensino Superior(Teresina-PI). Email: sarahnilkece@hotmail.com. 2 Enfermeira. Doutora em Enfermagem. Professora do Programa de Pós-graduação em Enfermagem da UFPI.Teresina-PI.

3 Enfermeira. Doutora em Enfermagem. Professora do Programa de Pós-graduação em Enfermagem da UFPI. Presidente do Conselho Regional de Enfermagem do Piauí-COREN-PI.Teresina-PI. 4 Enfermeira. Doutora em Enfermagem. Professora do Programa de Pós-graduação em Enfermagem da UFPI.Teresina-PI.

5 Enfermeira. Mestre em Enfermagem pela UFPI. Enfermeira da ESF. Teresina-PI.

6 Enfermeira graduada pela UFPI.Teresina-PI.

*Trabalho financiado pelo Programa Institucional de Bolsas de Iniciação Científica (PIBIC). Apresentado no XIX Seminário de Iniciação Científica e II Seminário em Desenvolvimento Tecnológico e Inovação da Universidade Federal do Piauí (UFPI). Teresina (PI), 2010. 
Consideramos que pesquisar conhecimentos e práticas de enfermeiros da ESF sobre a obesidade infantil é relevante para que se possa delinear como esse distúrbio da infância se apresenta na atenção básica, contribuindo para a reflexão sobre as políticas públicas na área e para a melhoria da qualidade das práticas de saúde dirigidas às crianças e à comunidade. Nessa conjuntura, o presente estudo objetivou analisar os conhecimentos e práticas de enfermeiros da atenção básica sobre a obesidade infantil.

\section{METODOLOGIA}

Estudo exploratório descritivo com abordagem quantitativa cujo cenário foram os centros de saúde da ESF da zona urbana da área de abrangência da Regional Leste-Sudeste de Teresina-PI, constituída por 23 centros de saúde, perfazendo um total de 68 equipes da ESF. A população foi constituída por enfermeiros atuantes em $50 \%$ das equipes da ESF que atuam na supracitada zona, configurando uma amostra de 34 destes profissionais, obtida por amostragem probabilística aleatória simples. A coleta de dados ocorreu em fevereiro e março de 2010 por meio de aplicação de formulário estruturado, mediante o consentimento expresso da população estudada, pela assinatura do Termo de Consentimento Livre e Esclarecido.

Para inclusão dos participantes foram considerados os seguintes critérios: ser enfermeiro, atuar na ESF da área de abrangência da pesquisa, desempenhar consultas de enfermagem em puericultura na estratégia.

Em obediência a todos os aspectos contidos na resolução 196/96 (7), a pesquisa foi realizada após aprovação pelo Comitê de Ética em Pesquisa da Universidade Federal do Piauí sob o n. 0171.0.045.000-09.

\section{RESULTADOS E DISCUSSÃO}

Na Tabela 1, apresentamos a caracterização dos 34 enfermeiros participantes do estudo.

Tabela 1 - Caracterização profissional dos enfermeiros do estudo. Teresina, 2010.

\begin{tabular}{|c|c|c|}
\hline VARIÁVEIS & $\mathbf{N}$ & $\%$ \\
\hline \multicolumn{3}{|l|}{ Titulação } \\
\hline Especialização & 27 & 79,5 \\
\hline Graduação & 5 & 14,7 \\
\hline Mestrado & 1 & 2,9 \\
\hline Doutorado & 1 & 2,9 \\
\hline \multicolumn{3}{|c|}{ Tempo de trabalho na Estratégia de Saúde da Família (ESF) } \\
\hline $0-4$ anos & 7 & 20,6 \\
\hline $5-9$ anos & 17 & 50 \\
\hline 10-14 anos & 8 & 23,5 \\
\hline 15 anos ou mais & 2 & 5,9 \\
\hline \multicolumn{3}{|c|}{ 3. Treinamento específico em Saúde Infantil } \\
\hline Sim & 21 & 61,8 \\
\hline Não & 13 & 38,2 \\
\hline \multicolumn{3}{|c|}{ Treinamento específico em Nutrição e Dietética } \\
\hline Sim & 7 & 20,6 \\
\hline Não & 27 & 79,4 \\
\hline
\end{tabular}

Dos 34 enfermeiros participantes do estudo, a expressiva maioria $(79,5 \%)$ tinha a especialização lato sensu como sua maior titulação e, em relação ao tempo de trabalho na ESF, o maior percentual (50\%) possuía de 5 a 9 anos de atuação. No tocante à capacitação em saúde infantil e nutrição, $61,8 \%$ afirmaram ter formação na área da saúde da criança, no entanto, $79,4 \%$ declararam não possuir treinamento específico em nutrição e dietética (Tabela 1).

Neste estudo, em que o enfoque se dá na saúde da criança, é motivador observar que um percentual significativo de enfermeiros da ESF demonstra possuir domínio em puericultura, o que torna mais fácil a adesão destes às políticas de prevenção e controle de agravos típicos da infância. Contraditoriamente a isso, percebe-se que no tocante à detecção de problemas alimentares infantis, os profissionais questionados ainda consideram seus conhecimentos insipientes, visto que, para promover um correto diagnóstico de enfermagem para obesidade infantil, orientações e manejo calórico, são necessários subsídios não só em saúde da criança, mas também em nutrição e dietética.

O enfermeiro da ESF deve ir além dos processos nutricionais. Deve estar preparado para aquilatar a influência dos fatores dietéticos externos determinados pelo grupo sobre a criança, que, por sua vez, contribuem fortemente para a apresentação e manutenção dos distúrbios metabólicos infantis ${ }^{(8)}$.

Tabela 2 - Distribuição dos enfermeiros segundo a adoção de estratégias de prevenção e detecção da obesidade infantil. Teresina-PI - 2010.

\begin{tabular}{|c|c|c|}
\hline VARIÁVEIS & $\mathbf{N}$ & $\%$ \\
\hline \multicolumn{3}{|l|}{ 1. Estratégias de prevenção da obesidade infantil } \\
\hline $\begin{array}{l}\text { Realizar avaliação antropométrica } \\
\text { da criança a cada consulta }\end{array}$ & 16,0 & 47,1 \\
\hline $\begin{array}{l}\text { Incentivar a dieta saudável e a prática } \\
\text { de atividade física }\end{array}$ & 15,0 & 44,1 \\
\hline Agendar visitas domiciliares & 3,0 & 8,8 \\
\hline \multicolumn{3}{|l|}{ 1. Uso do IMC para identificar a obesidade infantil } \\
\hline Sim & 30,0 & 88,2 \\
\hline Não & 4,0 & 11,8 \\
\hline \multicolumn{3}{|c|}{$\begin{array}{l}\text { 2. Condutas adotadas após identificação da obesidade } \\
\text { infantil }\end{array}$} \\
\hline $\begin{array}{l}\text { Realizar consultas de enfermagem } \\
\text { e encaminhar ao nutricionista }\end{array}$ & 20,0 & 58,8 \\
\hline Encaminhar ao nutricionista & 6,0 & 11,8 \\
\hline Encaminhar ao médico & 4,0 & 17,6 \\
\hline Planejar os cuidados multiprofissionalmente & 4,0 & 11,8 \\
\hline Realizar consultas de enfermagem isoladas & - & - \\
\hline
\end{tabular}

Em relação às estratégias de prevenção da obesidade infantil mais adotadas pelos enfermeiros da ESF, destaca-se que $47 \%$ destes costumam realizar avaliação antropométrica da criança a cada consulta de enfermagem; $44,1 \%$ se concentram em 
recomendar práticas saudáveis e $8,8 \%$ dos enfermeiros frequentemente agendam visitas periódicas aos domicílios para investigar a alimentação infantil, como maneira de evitar novos casos (Tabela 2).

Observa-se que a maioria dos enfermeiros recorre à avaliação antropométrica intramuros como principal método preventivo da obesidade infantil, no entanto, quando se trata da avaliação da criança em seu lócus, através das visitas domiciliares, os dados apontam para uma minoria engajada. Essa conclusão é preocupante quando se entende a obesidade infantil como uma afecção multifatorial, em que sua prevenção se torna mais eficaz quando o profissional se apropria do contexto no qual a criança está inserida.

Desta forma, entende-se que o investimento na prevenção da obesidade infantil nas primeiras etapas de vida, através da detecção de fatores de risco é capaz de evitar consequências a curto e longo prazos, como a incidência de coronariopatias e dislipidemias na vida adulta ${ }^{(9)}$. A promoção da saúde e a prevenção de agravos são os principais componentes de políticas de vida saudável para crianças, que podem ser fomentados pelo enfermeiro da $\operatorname{ESF}^{(10-11)}$.

Em relação ao conhecimento das formas de detecção da obesidade infantil, atestou-se que um número significativo de enfermeiros (88,2\%) utiliza o Índice de Massa Corpórea (IMC) como instrumento de detecção da obesidade infantil (Tabela 3).

$\mathrm{O}$ uso do IMC em crianças não é obrigatório para avaliar a composição da massa corpórea, visto que elas não apresentam hipertrofia muscular, o que pode mascarar ou determinar um possível excesso de peso. Desta forma, indica-se na ESF o uso das curvas antropométricas presentes na caderneta da criança $^{(5)}$.

É importante observar que, mesmo considerando-se o IMC um índice pouco fidedigno para diagnosticar a obesidade infantil, ele é o método de escolha dos enfermeiros da ESF. Esse fato pode ser justificado pela carência de conhecimentos na área por parte dos profissionais, insipiência de capacitações e atualizações na temática ou ainda se pode atribuir à pouca abrangência de faixas etárias nas curvas antropométricas da caderneta da criança, em que só a faixa de 0 a 2 anos é contemplada, dificultando a delimitação de um perfil calórico dos infantes atendidos na atenção básica.

Após identificar a obesidade infantil, a maioria $(58,8 \%)$ dos enfermeiros afirmou fazer o seguimento da criança pela consulta de enfermagem e, em seguida, encaminhar ao nutricionista. $17,6 \%$ referenciam direto para o nutricionista; $11,8 \%$ admitiram encaminhar ao médico e igual percentual afirmou expor o problema da criança para a equipe e planejar os cuidados multiprofissionalmente. No entanto, nenhum enfermeiro relatou fazer o seguimento da criança sem a ajuda de outros profissionais (Tabela 2).

O que se pode analisar com esses dados é que a ideia de assistência multiprofissional, proposta pelas atuais diretrizes em saúde, parece estar se interiorizando no profissional da atenção básica. Os resultados demonstram que boa parte dos enfermeiros da ESF vem compartilhando a responsabilidade do cuidado com sua equipe, principalmente quando o sujeito foco do cuidado é a criança, ser de tantas peculiaridades. Entender de forma holística o universo da criança obesa é imprescindível para amenizar não só as consequências fisiológicas da afecção, como também as repercussões psíquicas, como o bullying nas escolas.

A formação de uma rede de apoio à criança e à família que envolva artifícios lúdicos e que insira a escola como colaboradora do processo terapêutico infantil são medidas alternativas que fomentam a adesão da criança ao tratamento e fortalecem sua resolubilidade.

Assim, entende-se que o cuidado e a promoção da saúde infantil devem ser planejados na perspectiva holística de visões interdisciplinares. Profissionais que trabalham com a criança obesa devem buscar a autorreflexão sobre os próprios conceitos e conhecimentos a respeito da obesidade, o que proporciona maior capacidade para a ação(12)

Entretanto, há que se destacar que uma parcela dos enfermeiros arguidos, ainda não conscientes de suas competências na promoção e prevenção da saúde da criança na ESF, mostrou se eximir de sua função, mantendo-se distantes da problemática, delegando diretamente a responsabilidade do cuidar da criança aos outros profissionais da equipe, como nutricionistas e médicos. Essa observação denota negligência destes profissionais, quando se considera a criança um ser com alto déficit do autocuidado ${ }^{(13)}$, sendo necessária a intervenção do enfermeiro e da família para potencializar seu healing ${ }^{(14)}$.

Tabela 3 - Distribuição do número de crianças com obesidade por área da ESF Leste-Sudeste e os fatores que mais interferem na assistência de enfermagem. Teresina-PI, 2010.

VARIÁVEIS
\begin{tabular}{|l|c|c|} 
1. Crianças obesas por área de atuação do enfermeiro \\
\hline 0-4 crianças & 19 & 55,8 \\
\hline 5-9 crianças & 13 & 38,2 \\
\hline 10 ou mais crianças & 2 & 5,9 \\
\hline \multicolumn{3}{|c|}{} \\
2. Fatores intervenientes ao cuidar da obesidade infantil \\
\hline Ausência de treinamento específico & 15 & 44,1 \\
\hline Tempo reduzido & 1 & 2,9 \\
\hline Não identificação com a saúde da criança & 1 & 2,9 \\
\hline É competência de outro profissional & 1 & 2,9 \\
\hline Não há dificuldades & 13 & 38,2 \\
\hline Outros fatores & 3 & 8,8 \\
\hline
\end{tabular}

Todos os enfermeiros questionados souberam informar o número de crianças com obesidade infantil em sua área de atuação da ESF. 55,8\% dos profissionais relataram existir de 0-4 crianças obesas. Na área de atuação de $38,2 \%$ dos enfermeiros constavam de 5-9 crianças com obesidade infantil, enquanto há 10 ou mais crianças obesas na área de 5,9\% dos enfermeiros (Tabela 3).

Particularmente à área de atuação de cada enfermeiro 
entrevistado da ESF, o fator apontado que mais dificulta no manejo das crianças obesas é a falta de treinamento específico para lidar com o distúrbio (44,1\%). 2,9\% afirmaram que o maior empecilho é o tempo reduzido, por conta das diversas atribuições na unidade básica. Percentual igual admitiu não se identificar com a saúde da criança e afirmou ser responsabilidade de outros profissionais da equipe. Entretanto, $38,2 \%$ dos profissionais relataram não haver dificuldades para lidar com a obesidade infantil e $8,8 \%$ apontaram outros fatores, como a falta de experiência na área e carência de recursos físicos nas unidades de saúde (Tabela 3).

Pode-se perceber que os enfermeiros da ESF têm dificuldade de realizar a tomada de decisão clínica ou mesmo compor um raciocínio diagnóstico de obesidade infantil por julgarem seu conhecimento insuficiente. $\mathrm{O}$ empowerment ${ }^{(15)}$ (emponderamento) do profissional se dá pelo fortalecimento de sua autoconfiança, alcançada por capacitações e apreensão de um conhecimento que possa fundamentar sua prática. Transpor obstáculos, como o despreparo técnico e a excessiva burocratização imposta pelos sistemas de saúde, que exaure o tempo que poderia ser melhor despendido na assistência, deve ser objetivo precípuo do enfermeiro.

Na década de 1980, no Brasil, foi elaborado o programa de "Assistência Integral à Saúde da Criança", no entanto, dentro dessa estratégia não há um plano de ação específico voltado para a obesidade infantil(10). Um dos problemas prevalentes na ESF é a execução de uma atenção primária reducionista, em decorrência de baixa qualificação profissional, despreparo técnico, reduzido número de trabalhadores ou pequeno investimento político na atenção primária à saúde, o que leva a não efetivação das ações básicas ${ }^{(16)}$.

A despeito dos fatores intervenientes, é mister o engajamento do enfermeiro, sujeito que melhor representa a atenção básica nessa problemática, a partir da reivindicação por uma política que ampare as crianças vítimas da obesidade, bem como norteie a conduta dos profissionais que se colocam na linha de frente da saúde pública, munindo-os de conhecimento e meios logísticos que assegurem uma melhor qualidade da assistência a essas crianças. Investir em saúde da criança é pensar a longo prazo, visto que o sujeito, quando adulto, é consequência do que foi quando criança ${ }^{(17)}$.

\section{CONCLUSÃO}

A carência de educação continuada em enfermagem na atenção básica dificulta o vínculo da cadeia multiprofissional do cuidado, com o consequente isolamento do enfermeiro na tomada de decisões clínicas. Profissionais preparados e que façam a ponte entre os serviços de assistência, comunidade e família podem atingir resultados mais otimistas no manejo da criança obesa. Para tanto, faz-se necessário o investimento em programas de educação continuada para os enfermeiros da ESF, como forma de viabilizar a resolubilidade dos problemas da comunidade em nível básico, reduzindo a demanda do usuário a estratos mais complexos de atendimento.

Políticas públicas de atenção à saúde da criança na ESF, que enfoquem a obesidade infantil, são essenciais para nortear as condutas adotadas. Para tanto, acrescenta-se a necessidade de um estudo mais amplo nessa perspectiva, a fim de se diagnosticar essa situação vivenciada para fundamentar a adoção destas políticas.

\section{Referências}

1. Oliveira AMA, Cerqueira EMM, Souza JS, César AO. Sobrepeso e obesidade

infantil: influência de fatores biológicos e ambientais da Feira de Santana-BA.

Arq Bras Endocrinol Metab. 2003;47(2):144-50.

2. Styne DM. Childhood and adolescent obesity - Prevalence and

significance. Pediat Clin North Amer. 2001;48:823-53.

3. Dietz WH. The obesity epidemic in young children. BMJ. 2001;322(7282):313-4.

4. Oliveira CL, Fisberg M. Obesidade e adolescência na infância: uma verdadeira

epidemia. Arq Bras Endocrinol Metab. 2003;47(2):107-8.

5. Bernardes AC, Pimenta LP, Caputo ME. Obesidade infantil:correlação colesterol

e relação cintura-quadril. Juiz de Fora: Universidade Gama Filho; 2000.

6. Marcondes E, Vaz FAC, Okay Y, Ramos JLA. Pediatria básica. 9a ed. São Paulo:

Sarvier; 2002

7. Ministério da Saúde (BR). Conselho Nacional de Saúde. Comissão Nacional

de Ética em Pesquisa. Resolução no 196 de 10 de outubro de 1996: diretrizes

e normas regulamentadoras de pesquisa envolvendo seres humanos. Brasília:

Ministério da Saúde; 1996.

8. Figueiredo GLA, Mello DF. A prática da enfermagem na atenção à saúde da

criança na Unidade Básica de Saúde. Rev Latinoam Enferm. 2003;11(4):544-51.

9. Boog MCF. Dificuldades encontradas por médicos e enfermeiros na abordagem de problemas alimentares. Rev Nutr. 1999;12(3):261-72.

10. Oliveira AMA, Cerqueira EMM, Oliveira AC. Prevalência de sobrepeso e

obesidade infantil na Cidade de Feira de Santana-BA: detecção na Família x

Diagnóstico Clínico. J Pediatr. 2003;79(4):325-8.

11. Tomkins A. Que padrões usar para medir obesidade em crianças?. J Pediatr.

2006;82(4):246-8.

12. Sabia RV, Santos JE, Ribeiro RPP. Efeito da atividade física associada à

orientação alimentar nos adolescentes obesos: comparação entre o exercício

aeróbio e anaeróbio. Rev Bras Med Esporte. 2004;10(5):349-55.

13. Mcewen $M$, Wills EM. Bases teóricas para a enfermagem. $2^{\mathrm{a}}$ ed. Porto Alegre:

Artmed; 2009.

14. Watson J. Caring science as sacred science. Philadelphia: FA. Davis; 2005.

15. Carvalho SR. Os múltiplos sentidos da categoria "empowerment" no projeto

de Promoção à Saúde. Cad Saúde Pública. 2004;20(4):1088-95.

16. Sucupira AC. Representando a atenção à saúde da criança e do adolescente

na perspectiva intersetorial. Rev Adm Pública. 1998;32(2):61-78.

17. Frota MA, Maia JA, Pereira AS, Nobre CS, Vieira LJES. Reflexão sobre

políticas públicas e estratégias na saúde integral da criança. Enferm Foco.

2010;1(3):129-32. 